\title{
AN INEQUALITY FOR A PAIR OF POLYNOMIALS THAT ARE RELATIVELY PRIME
}

\author{
K. MAHLER \\ (received 28 February 1964) \\ To T. M. Cherry
}

Let $f(x)$ and $g(x)$ be two polynomials with arbitrary complex coefficients that are relatively prime. Hence the maximum

$$
m(x)=\max (|f(x)|,|g(x)|)
$$

is positive for all complex $x$. Since $m(x)$ is continuous and tends to infinity with $|x|$, the quantity

$$
E(f, g)=\min _{*} m(x)
$$

is therefore also positive.

In the theory of transcendental numbers one often requires a good positive estimate for $E(f, g)$. The usual method for obtaining such an estimate is as follows. If $R(f, g)$ denotes the resultant of $f$ and $g$, then identically in $x$

$$
f(x) F(x)+g(x) G(x)=R(f, g)
$$

where $F(x)$ and $G(x)$ are two polynomials that can be defined explicitly in terms of determinants. It follows that

$$
m(x) \geqq|R(f, g)| /\{|F(x)|+|G(x)|\},
$$

and hence it suffices to give an upper estimate for $|F(x)|+|G(x)|$. For this purpose one may assume that $|x|$ is not too large; for when $|x|$ is large, $m(x)$ trivially cannot be small. (See e.g. A. O. Gelfond, Transcendentnye i algebraitcheskie tchisla, Moskva 1952, pp. 181-2.)

In the present note I shall apply a different and better method that is due to N. Feldman. It has the additional advantage of leading to a bestpossible result.

1. Let, in explicit form,

$$
f(x)=a_{0}\left(x-\alpha_{1}\right) \cdots\left(x-\alpha_{m}\right), \quad g(x)=b_{0}\left(x-\beta_{1}\right) \cdots\left(x-\beta_{n}\right),
$$

where $a_{0} \neq 0$ and $b_{0} \neq 0$, and where $\alpha_{k} \neq \beta_{k}$ for all $h$ and $k$. Put, for any given complex number $x$, 


$$
\alpha=\min _{1 \leqq n \leqq m}\left|x-\alpha_{n}\right| \text { and } \beta=\min _{1 \leqq k \leqq n}\left|x-\beta_{k}\right| \text {, }
$$

and denote by $r$ and $s$ two suffixes for which

$$
\alpha=\left|x-\alpha_{r}\right| \text { and } \beta=\left|x-\beta_{s}\right| \text {. }
$$

Then at least one of the two numbers $\alpha$ and $\beta$ is positive.

Assume, first, that

$$
0<\alpha \leqq \beta
$$

and number the zeros of $g(x)$ such that, say,

$$
\left|\alpha_{\mathrm{r}}-\beta_{k}\right| \begin{cases}<2 \alpha & \text { if } \quad k=1,2, \cdots, N, \\ \geqq 2 \alpha & \text { if } \quad k=N+1, N+2, \cdots, n ;\end{cases}
$$

here $N$ is a certain integer satisfying $0 \leqq N \leqq n$.

If $k=1,2, \cdots, N$, then

$$
\left|x-\beta_{k}\right| \geqq \beta \geqq \alpha>\left|\alpha_{r}-\beta_{k}\right| / 2 .
$$

If, however, $k=N+1, N+2, \cdots, n$, then

and therefore

$$
\left|\alpha_{r}-\beta_{k}\right| \geqq 2 \alpha=2\left|x-\alpha_{r}\right|
$$

$$
\left|x-\beta_{k}\right|=\left|\left(x-\alpha_{r}\right)+\left(\alpha_{r}-\beta_{k}\right)\right| \geqq\left|\alpha_{r}-\beta_{k}\right|-\left|x-\alpha_{r}\right| \geqq\left|\alpha_{r}-\beta_{k}\right| / 2 .
$$

On combining the inequalities (1) and (2) it follows that

$$
|g(x)|=\left|b_{0} \prod_{k=1}^{n}\left(x-\beta_{k}\right)\right| \geqq 2^{-n}\left|b_{0} \prod_{k=1}^{n}\left(\alpha_{r}-\beta_{k}\right)\right| .
$$

It is obvious that this formula remains true also when

$$
\alpha=0,
$$

and hence we have proved that

$$
|g(x)| \geqq 2^{-n}\left|g\left(\alpha_{r}\right)\right| \text { if } \quad 0 \leqq \alpha \leqq \beta .
$$

In exactly the same way it follows that

$$
|f(x)| \geqq 2^{-m}\left|f\left(\beta_{s}\right)\right| \text { if } \quad 0 \leqq \beta \leqq \alpha .
$$

These two inequalities together imply the following result.

THEOREM 1. Let $f(x)$ and $g(x)$ have the degrees $m$ and $n$ and the zeros $\alpha_{1}, \cdots, \alpha_{m}$ and $\beta_{1}, \cdots, \beta_{n}$, respectively. Then

$$
E(f, g) \geqq \min _{\substack{1 \leqq n \leqq m \\ 1 \leqq k \leqq n}}\left(2^{-m}\left|f\left(\beta_{k}\right)\right|, 2^{-n}\left|g\left(\alpha_{n}\right)\right|\right) .
$$


This result is best possible because the assertion holds with equality in the special case when $f$ and $g$ are the two polynomials

$$
f(x)=(x-1)^{m} \text { and } g(x)=(x+1)^{n} .
$$

2. Theorem 1 gives a lower bound for $E(f, g)$ in terms of the zeros of $f$ and $g$. It is now not difficult to replace this estimate by one that involves instead only the coefficients of these two polynomials.

Let in explicit form

$$
f(x)=a_{0} x^{m}+a_{1} x^{m-1}+\cdots+a_{m}, \quad g(x)=b_{0} x^{n}+b_{1} x^{n-1}+\cdots+b_{n} .
$$

Further denote by

$$
L(f)=\left|a_{0}\right|+\left|a_{1}\right|+\cdots+\left|a_{m}\right|, \quad L(g)=\left|b_{0}\right|+\left|b_{1}\right|+\cdots+\left|b_{n}\right|
$$

the lengths of the two polynomials. By a theorem of R. Güting ${ }^{1}$,

$$
\left|f\left(\beta_{k}\right)\right| \geqq|R(f, g)|\left|L(f)^{n-1} L(g)^{m}, \quad\right| g\left(\alpha_{n}\right)|\geqq| R(f, g)|| L(f)^{n} L(g)^{m-1}
$$

for all suffixes $h$ and $k$. Hence, by Theorem 1 ,

$$
E(f, g) \geqq|R(f, g)| L(f)^{-n} L(g)^{-m} \min \left\{2^{-m} L(f), 2^{-n} L(g)\right\} .
$$

For the applications, the most important case is that of polynomials with integral coefficients. The resultant $R(f, g) \neq 0$ is then also an integer and hence its absolute value is not less than 1 . Therefore, in this particular case,

$$
E(f, g) \geqq L(f)^{-n} L(g)^{-m} \min \left\{2^{-m} L(f), 2^{-n} L(g)\right\} .
$$

While this formula is very simple, it is, however, no longer best possible.

Canberra, 19 February, 1964.

Mathematics Department, IAS,

Australian National University.

1 Approximation of algebraic numbers by algebraic numbers, Michigan Math. J. 8 (1961), 149-159. 